Aus der chirurgischen Poliklinik Basel (Direktor: Professor de Quervain).

\title{
Wachstumsbeschwerden zur Zeit der knöchernen Entwicklung der Tuberositas metatarsi quinti.
}

\author{
Von Dr. Hans Iselin, P. D. leitender Arzt.
}

(Mit 7 Abbildungen.)

Die fibulare Epiphyse der Tuberositas metatarsi $V$ tritt im Alter von I $3-I_{5}$ Jahren als cin konstantes Gebilde auf ${ }^{1}$ ). Diesen Knochenkern habe ich nach Wenzel Gruber, der ihn zuerst beschrieben hat, genannt. Die Arbeiten von A. Kirchner ${ }^{2}$ ) waren mir bei meiner damaligen Veröffentlichung nicht bekannt. Kirchner schildert 6 Mittelfußknochen $V$, an denen entweder verknöcherte, aber noch nicht verschmolzene distale Epiphysen vorhanden sind, oder vorhanden gewesen, oder abgefallen sind ${ }^{3}$ ). Kirchner hat auch einige Röntgenaufnahmen bei II-I6Jährigen gemacht. I)iesc zeigten nicht regelmäßig eine proximale Epiphyse am Metatarsale V. Bei einem $13^{1 / 2}$ Jährigen war sie sehr deutlich. „Wie oft sie vorkommt, ob sie vielleicht konstant oder annähernd konstant, dazu bedarf es einer größern Anzahl von Beobachtungen, welche $\mathrm{zu}$ machen besonders die Chirurgen ausgiebige Gelegenheit haben," schreibt Kirchner ${ }^{4}$ ). Diese Ergänzung meiner ersten Mitteilung ist mir eine angenehme Anstandspflicht. Die Befunde bestätigen sich durch gute Übereinstimmung. Um die Stetigkeit des Auftretens in diesem Alter außer Zweifel zu setzen, untersuchte mein Assistent, Dr. Schou-

1) I s e I in, Die Wenzel-Grubersche fibulare Epiphyse der Tuberositas metatarsi quinti im Röntgenbild. Deutsche Zeitschr. f. Chir., Bd. 92, S. 56I.

2) Die Epiphyse am proximalen Ende des Os metatarsi $V$, aus den anatomischen Heften, Bd. 33, H. I01, S. 15, und Arch. f. klin. Chir., Bd. 8o, S. 719 .

3) Anatomisches Heft, S. 518 .

4) Langenbecks Archiv, S. 722. 
wey, an dem Röntgenbild der gleichen von mir damals photographierten 6o Füße, ob seinerzeit fehlende Kerne inzwischen aufgetreten sind, und gleichzeitig sucht er zu bestimmen, wie rasch die Apophysen mit dem Knochen verwachsen.

Der Zweck dieser Mitteilung ist, das Bild einer Krankheit wiederzugeben, die sich an der Tuberositas metatarsi $V$ abspielt und genau den Erscheinungen der sog. Schlatterschen Krankheit an der Tuberositas tibiae entspricht. Dieser Befund ist noch nicht beschrieben worden.

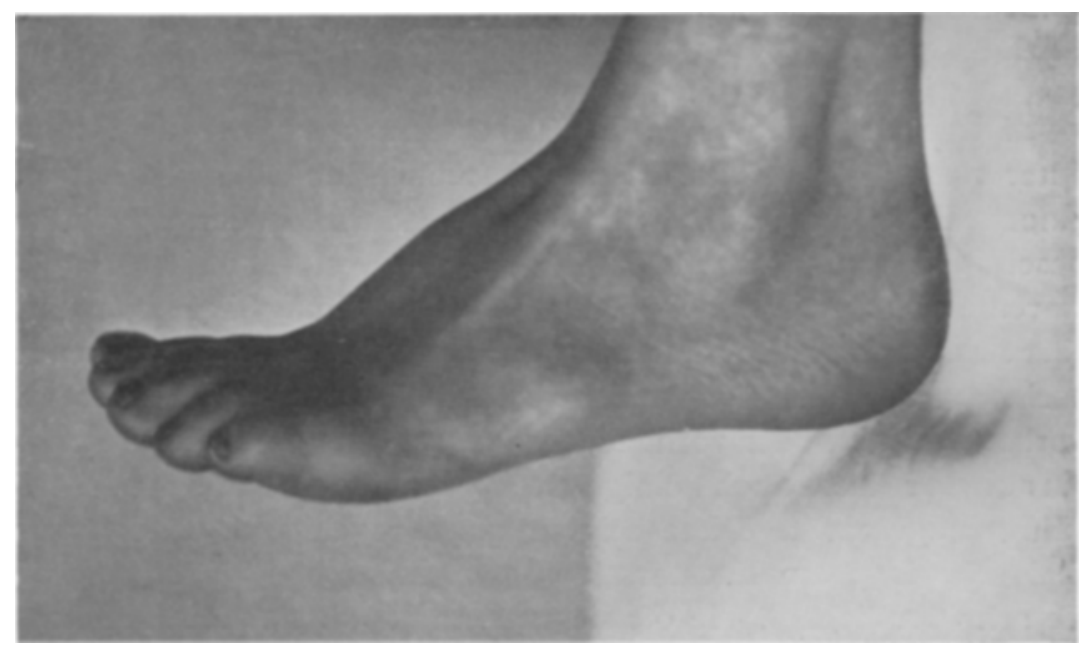

Fig. I. Schwellung der Tuberositas metatarsi $V$ (Wachstumsbeschwerden).

Ein 13 jährigcs Mädchen holte sich in der chirurgischen Poliklinik 1909 Rat wegen einer schmerzhaften Anschwellung im Bereich der Tuberositas des V. Mittelfußknochens. Die Schwellung war druckempfindlich, beim Gehen schmerzhaft, sie fühlte sich derb an, zeigte aber weder (Odem noch Rötung. (Vgl. die photographische Aufnahme Fig. I.) Auf der andern Seite fehlte diese Schwellung. Im Röntgenbild ist auf der kranken Seite der beschriebene Knochenkern größer als auf der gesunden (Fig. 2). Stärkere Veränderungen fehlten, namentlich war keine Verletzung nachzuweisen. Die Kranke und auch ihre Mutter konnten sich nicht erinnern, daß das Kind sich irgendwie 
oder irgendwann verletzt hatte. Auch für häufige kleinere Verletzungsarten war kein Anhaltspunkt in der Krankengeschichte. Patientin machte keine besondern körperlichen Anstrengungen, sie huldigte nicht einmal dem üblichen Vergnügen des Seilspringens. Das Kind war ziemlich gut entwickelt.

Was das stoffliche Wesen der Erkrankung ausmacht, ist noch nicht ermittelt. Für die Tuberositas tibiae hat 1899 Aboulker ${ }^{1}$ ) eine traumatische Knochenentzündung angenommen, während Schlatter $1903^{2}$ ) die Erscheinungen durch

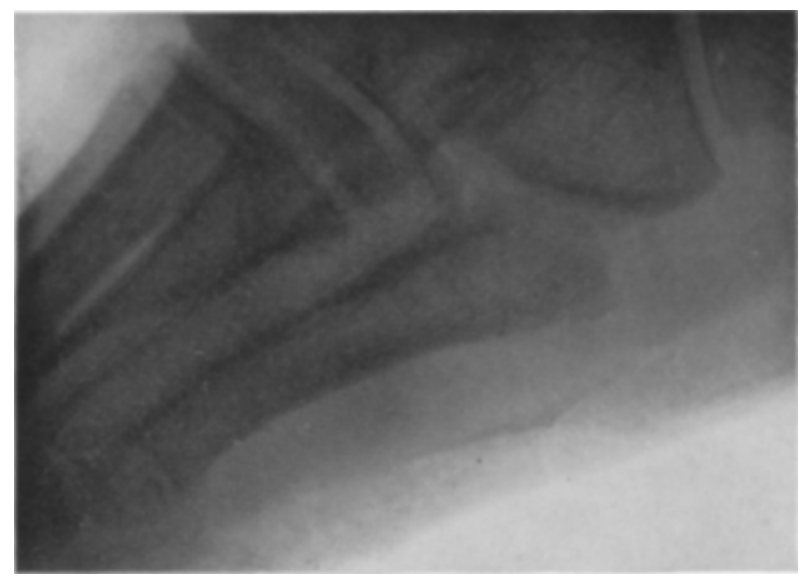

Fig. 2. Tuberositas metatarsi $\mathrm{V}$ mit Knochenkern Röntgenbild von Figur I.

das Vorhandensein einer unvollständigen Fraktur erklären wollte. Da Schlatter als erster in deutscher Sprache diese Veränderung bei jugendlichen Individuen besprochen hat, ist die Schlattersche Fraktur im Laufe der Jahre, weil sich die Auffassung geändert hat, Schlatter zu Ehren in die sog. Schlattersche Krankheit umgetauft worden.

I) De l'ostéite apophysaire du tibia pendant la croissance. Thèse de Lyon.

2) S ch latter, Verletzungen des schnabelförmigen Fortsatzes der oberen Tibiaepiphyse. Bruns' Beiträge Bd. 38, S. 874. Später I9o8 Schlatter: Unvollständige Abrißfrakturen der Tuberositas tibiae oder Wachstumsanomalien? Bruns' Bd. 59, S. 518 . 
Hier, wie bei der entsprechenden Erkrankung am Knic, fehlen zu einer eigentlichen Entzündung die lokale Temperaturerhöhung und auch die entzündliche Weichteilschwellung. Vielleicht handelt es sich um eine allgemeine Hypertrophie der 'Teile, die mit der rascheren Bildung der knöchernen Ansatzstelle einhergeht, also um einen beschleunigten Bildungsvorgang, der schmerzhaft ist. Kleinere Verletzungen sind nicht ganz auszuschließen. Ciese dürften aber am Knie nur durch direkte Gewalt, Fall aufs Knic, entstehen, nicht aber als Abrißfrakturen, wie das
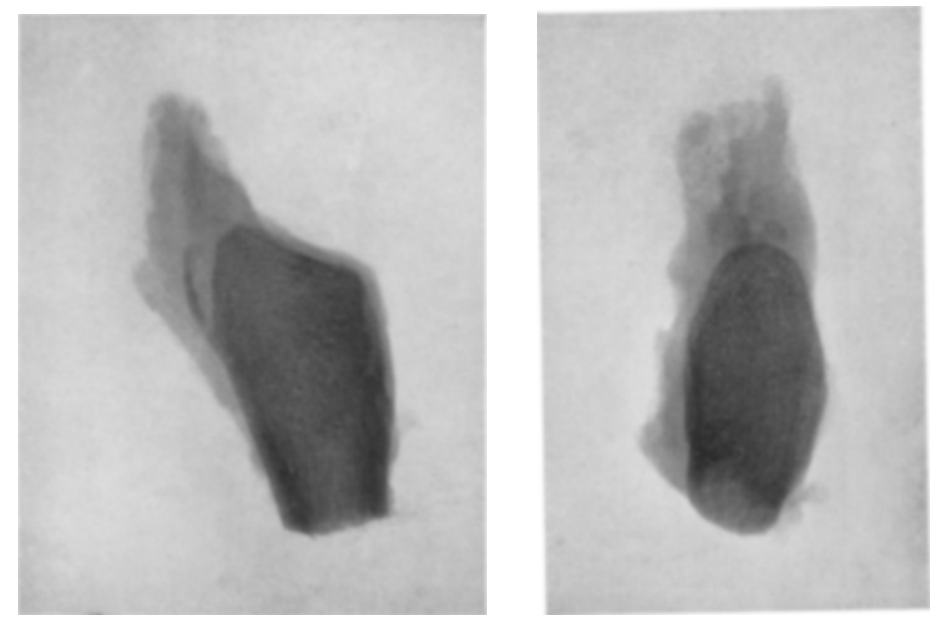

Fig. 3. Röntgenbild der Tuberositas metatarsi $\mathrm{V}$ mit Peronäusschne (frisches anatomisches Präparat). Der Knochenkern entspricht dem Sehnenansatz.

Schlatter annahm, aufgefaßt werden, da der Knochenkern direkt in die Sehne cingeschaltet ist und der ganze zungenförmige Fortsatz oder Rüssel der Tibiaepiphyse durch ein festes Band, das federnde Bewegungen gestattet, an der Tibia befestigt ist, also kaum größere Abrisse zuläßt ${ }^{1}$ ). Diese Verhältnisse konnte ich durch anatomische und histologische Lntersuchung feststellen.

Auch an der 'Tuberositas metatarsi $V$ steht der Knochenkern in enger Verbindung mit der Sehne selbst; er scheint, wie das

I) Es ist möglich, daßß ein einzelnes kleineres oder größeres Sehnenbünde! mit Knochensubstanz aus dem in der Sehne entstchenden Knochenkern ausgerissen wird; mehrere meinel Röntgenbilder machen es wahrscheinlich, daL 
Röntgenbild eines ausgeschälten Metatars V (Fig. 3) von einem I 5 jährigen Jungen zeigt, welcher durch einen Hirnschuß umgekommen war, mit der Sehne des Peroneus brevis der knorpelig vorgebildeten Tuberositas metatarsi $\mathrm{V}$ aufzusitzen. In dem histologischen Bild zichen die Sehnenfasern bis in den Knochenkern hinein, auf der andern konkaven Seite wandelt sich der Knorpel in Knochengewebe um.

Für eine Hypertrophie der Gewebe sprechen noch zwei Beobachtungen von meinem früheren Chef, Prof. Hildebrand. Er hat, als Schlatter die Behauptung aufstellte, daß es sich bei dieser schmerzhaften Anschwellung des vorderen Schienbeinhöckers um Frakturen handle, 2 mal bei solchen Frkrankten auf die Tuberositas eingeschnitten. Die längsgespaltene Sehne und das Periost schienen zwiebelschalenförmig verdickt; diese Gewebe waren mit Knochen durchsetzt, der den Eindruck einer Exostose machte. Von Trauma, alter Blutung usw. war nichts nachzuweisen. Leider fehlt in den Krankengeschichten der Befund der histologischen Untersuchung.

Eine genügende Erklärung für diese schmerzhafte Entwicklung der Apophyse können wir noch nicht geben.

Ähnliche Beschwerden können aber auch bei der Verschmelzung von eigentlichen Epiphysenkernen auftreten. Im Sommer I 9 IO wurde der Klinik, als ich stellvertretender Leiter war, ein I 5 jähriger Jüngling wegen Absprengung am Ellbogengelenk zur Operation zugeschickt. Diese Diagnose gründete sich nicht nur auf das Röntgenbild, sondern auch auf die Klagen des Knaben und den Palpationsbefund. Es bestand eine deutliche Schwellung in der Fossa olecrani; die Kapsel schien verdickt; die Gegend des Olecranon und auch die Kapsel waren druckempfindlich. Das Röntgenbild zeigte, daß der obere Knochenkern des Olecranons viel besser entwickelt war als auf der andern Seite. Er ist auf dem Bilde so isoliert, da $B$ es nahe lag, an eine Absprengung zu denken. Die Operation wurde nicht vorgenommen; der Junge

solche anfangs nur wenig verlagerte Knochenstückchen zu einem kuglichen Gebilde weiterwachsen und $\mathrm{da}$ dieses selbständige Knochenkörperchen mit der wachscnden Sehne sich von ihrem Ursprungsort immer mehr entfernt. Dieser Schaltknochen kann Beschwerden machen. Eine solche Versprengung von Knochensubstanz kann vielleicht auch ohne Verletzung zustande kommen. 


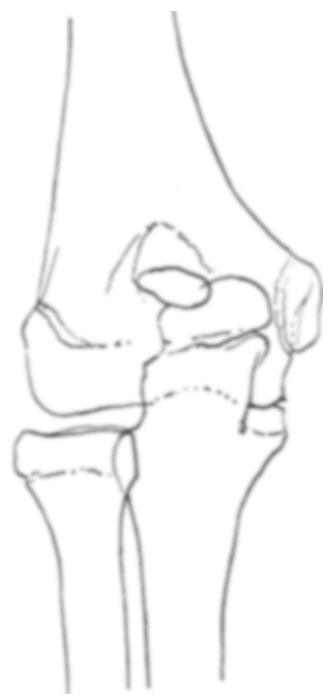

Fig. 4. Wachstumsbeschwerden bei der Entwicklung des 2. Knochenkernes im Olecranon. Als Gelenkkörper der Klinik eingewiesen.

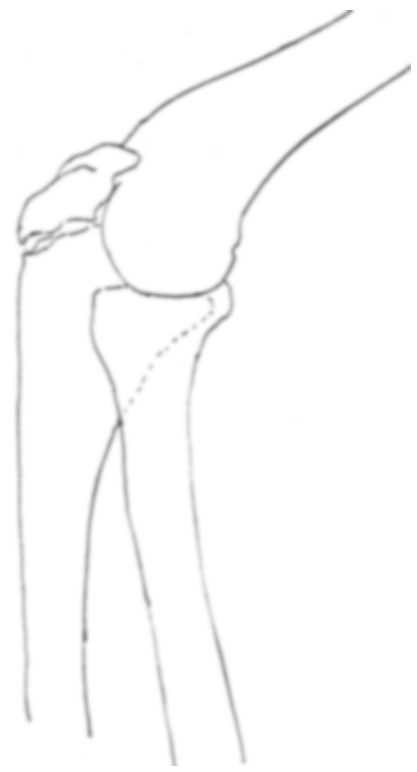

Fig. 6. Derselbe Ellenbogen wie in Figur 4, seitliche Aufnahme.

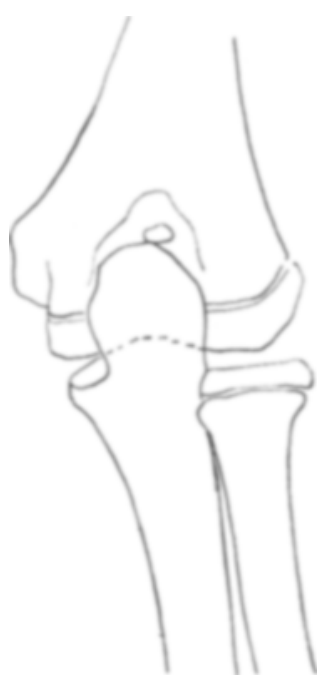

Fig. 5. Bild der gesunden Seite. Kern bedeutend kleiner mit langsamerer Entwicklung.

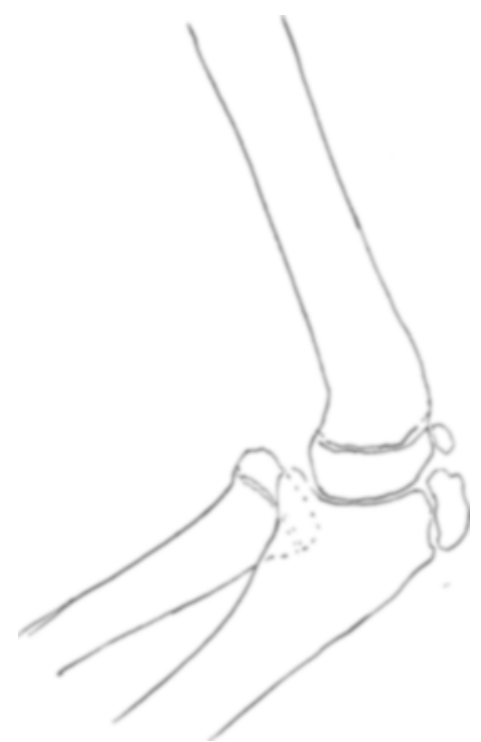

Fig. 7. Gesunde Seite. 
wurde entlassen, bei der $\Lambda$ rbeit noch eine Zeitlang geschont; jetzt arbeitet er wieder ohne Beschwerden. (Vgl. die beiden Röntgenbilder, Fig. 4, 5, 6, 7.)

Ob es angeht, die Schmerzen durch eine stärkere Dchnung des Periosts infolge der raschen Knochenentwicklung anzunehmen, scheint mir fraglich; immerhin ist der Gedanke doch erwägenswert.

Aus dem Mitgeteilten ist zu ersehen, daß die Entwicklung der Apophysen, d. h. die knöcherne Bildung der Höcker an Stellen, wo eine Sehne ansetzt, mit Schmerzen und Schwellung einhergehen kann, an der Tuberositas metatarsi $\mathrm{V}$ ganz gleich wie an der Tuberositas tibia; ohne daß Verletzungen vorangegangen sind. - Solche besondern Knochenkerne hat Verfasser im Röntgenbild nachweisen können, am Processus styloides ulnae, am Acromion und Coracoid, am Angulus scapulae, am Tuber ischii, an der Crista ilii und ausnahmsweise am Malleolus internus. Diese Knochenkerne, auch Apophysen genannt, waren den alten Anatomen Schwegel, Béclard, Rambaud, Renault und Köllicker ${ }^{1}$ ) sehr gut bekannt. Diese Autoren haben auch an der Tuberositas radii cinen besondern Knochenkern gefunden. Ein Finweis auf das Vorkommen dieser Apophysen ist angezeigt, da in den Röntgenatlanten Sicks, Wilms und Grasheys diese weniger auffälligen, meist konstanten Befunde gar nicht erwähnt sind, und diese Befunde von Ärzten, welche der Röntgenbilder unkundig sind, oft als Knochenbrüche gedeutet werden.

I) Anmerkung: Schwege l, Entwicklungsgeschichte der Knochen, des Stammes und der Extremität. Sitzungsbericht der Wiener Akademie der Wissenschaften, Bd. 30 aus dem Jahre 1858 . R a m b a ud und Renault, Orig. et développement des os; Paris 1864, S. 207. B éclard, Úber die Osteose oder die Bildung, das Wachstum und die Altersabnahme der Knochen des Menschen. Meckels Archiv ı820, Bd. 6. Köllicker, Entwicklungs. geschichte der Menschen und höheren Tiere I879, S. 50. 